\title{
PACKING AND REFLEXIVITY IN BANACH SPACES
}

BY

\author{
CLIFFORD A. KOTTMAN( $\left.{ }^{1}\right)$
}

\begin{abstract}
A measure of the "massiveness" of the unit ball of a Banach space is introduced in terms of an efficiency of the tightest packing of balls of equal size in the unit ball. This measure is computed for the $l_{p}$-spaces, and spaces with distinct measures are shown to be not nearly isometric. A new convexity condition, which is compared to $B$-convexity, uniform smoothness, and uniform convexity, is introduced in terms of this measure, and is shown to be a criterion of reflexivity. The property dual to this convexity condition is also exposed and examined.
\end{abstract}

0. Introduction. The technique of characterizing the "massiveness" of a subset of a metric space by means of most economical coverings or by densest packings of $\varepsilon$-neighborhoods is one frequently used. Recently, Robert Whitley [10] investigated properties of economical coverings of the unit sphere in a Banach space and obtained some interesting results about nearly isometric spaces. In this paper we introduce a measure of the efficiency of the tightest possible packings of equal balls within the unit sphere. This measure yields information similar to Whitley's results on nearly isometric spaces and has the added advantage of being a criterion for reflexivity.

We shall employ the following notation. For a normed linear space $X$, the unit ball will be denoted $U(X)=\{x \mid\|x\| \leqq 1\}$ and the unit sphere is $S(X)=\{x \mid\|x\|=1\}$. If $y$ is an element of $X$ and $r$ is a positive real number, the set $y+r U(X)$ is a translation and dilation of $U(X)$ and is called simply a ball of radius $r$.

Definition 0.1. For each cardinal number $\alpha$ let $P(\alpha, X)=\sup \{r \mid$ there exist $\alpha$ disjoint balls of radius $r$ in $U(X)\}$. (In this setting we take sup $\varnothing=0$.)

We develop in $\S 1$ the basic tools which are used throughout the remainder of the paper. We show that the number $P(\alpha, X)$ is closely related to $\varepsilon$-separated subsets of $S(X)$ (Theorem 1.3) and we find conditions on $\alpha$ that guarantee that $\frac{1}{3} \leqq P(\alpha, X)$ $\leqq \frac{1}{2}$ (Lemma 1.2). Finally we give an example that provides a partial converse to this last result (Example 1.6) and a counterexample to a likely-sounding conjecture (Example 1.8).

Presented to the Society, January 24, 1969; received by the editors January 6, 1969.

AMS Subject Classifications. Primary 4610, 5245; Secondary 5240, 4645.

Key Words and Phrases. Packing, reflexivity, nearly isometric spaces, $B$-convexity, $P$ convexity, uniformly smooth, uniformly convex, geometry of the unit ball.

(1) This work was supported by a National Science Foundation Graduate Fellowship, and represents a part of the author's Ph.D. thesis, which was submitted to the University of Iowa under the helpful direction of Professor Bor-Luh Lin.

Copyright (C) 1970, American Mathematical Society 
$\$ 2$ contains the result on nearly isometric spaces. It is shown that if for Banach spaces $X$ and $Y$ the numbers $P(\alpha, X)$ and $P(\alpha, Y)$ differ for some value of $\alpha$, then $X$ and $Y$ are not nearly isometric.

$\S 3$ relates the number $P(\alpha, X)$ to some work of Daniel Giesy [3] and R. C. James [5]. They have raised the question whether a particular geometric condition (called $B$-convexity) implies the reflexivity of every Banach space which satisfies it. Since this question has remained unanswered, it is natural to ask whether some slightly stronger geometric condition implies reflexivity. The concept of packing provides an appropriate vehicle to introduce the geometric condition $P$-convexity, which yields an affirmative answer to this question (Theorem 3.2). Furthermore, we show that some of the properties inherent in $B$-convexity are retained by $P$-convexity, and give an example to show that Theorem 3.2 cannot be extended in one direction.

$\S 4$ studies the property dual to $P$-convexity. This property is shown to be similar to $P$-convexity itself in several ways (Corollary 4.4 ).

It should also be noted that the packing number $P(\alpha, X)$ is related to the concepts of capacity and $\varepsilon$-capacity, which have been studied in detail by Kolmogorov [7] and others for certain subsets of function spaces.

A subset of a metric space is said to be $\varepsilon$-separated provided the distance between any two elements of the subset is at least $\varepsilon$. The density character of a metric space is the smallest cardinality that a dense subset may possess. A subset of a metric space is an $\varepsilon$-net, provided the distance from any point in the space to some point in the subset is at most $\varepsilon$.

1. Preliminaries. In this section we establish a relation between $P(\alpha, X)$ and $\varepsilon$-separated subsets of $S(X)$, which is the main tool for subsequent results, and illustrate its application with an example.

LEMMA 1.1. If $0<\varepsilon<1$ and $A$ is an $\varepsilon$-net for $S(X)$ with card $(A)=\alpha$, then the density character of $X$ is $\aleph_{0}$ if $\alpha$ is countable, and the density character of $X$ is less than or equal to $\alpha$ if $\alpha$ is uncountable.

Proof. Let $B$ be the set $\left\{\sum a_{i} x_{i}: a_{i}\right.$ rational and $x_{i}$ in $\left.A\right\}$, where the sums are finite. If $B$ is not dense in $X$, then $B$ is in a hyperplane through the origin and there exists $y$ in $S(X)$ with dist $(y, B)>\varepsilon$. Therefore $B$ is dense in $X$. Q.E.D.

We wish to thank the referee for simplifying the original proof of this lemma.

LEMMA 1.2. Let $X$ be an infinite dimensional normed space and $\alpha$ be a cardinal number greater than one but less than or equal to the density character of $X$. Then $\frac{1}{3} \leqq P(\alpha, X) \leqq \frac{1}{2}$.

Proof. It is clear that $P(\alpha, X)$ is decreasing for increasing values of $\alpha$, and that $P(2, X)=\frac{1}{2}$. Choosing $\alpha$ less than or equal to the density character of $X$, it remains to show that for each $\varepsilon>0$ there exist $\alpha$ disjoint balls of radius $\frac{1}{3}-\varepsilon$ in $U(X)$. Define $\mathscr{D}=\{A \subset S(X) \mid x, y \in A$ implies $\|x-y\|>1-\varepsilon\}$. Now if $\mathscr{D}$ is partially ordered by inclusion, Zorn's lemma applies and $\mathscr{D}$ has a maximal element $A_{0}$. 
Notice that $A_{0}$ forms a $(1-\varepsilon)$-net for $S(X)$. Hence by Lemma 1.1 card $\left(A_{0}\right)$ is greater than or equal to the density character of $X$, and so card $\left(A_{0}\right) \geqq \alpha$. Letting $B$ be a subset of $A_{0}$ of cardinality $\alpha$, the set $\left\{\frac{2}{3} y+\left(\frac{1}{3}-\varepsilon\right) U(X) \mid y \in B\right\}$ is the desired collection of disjoint balls of radius $\frac{1}{3}-\varepsilon$ contained in $U(X)$. Q.E.D.

THEOREM 1.3. If $\alpha$ is a cardinal number, $P(\alpha, X)=r>\frac{1}{3}$, and $\varepsilon>0$, then there is a $(2 r /(1-r)-\varepsilon)$-separated subset $A$ of $S(X)$ such that card $(A)=\alpha$ if $\alpha$ is infinite and card $(A) \geqq \alpha-1$ if $\alpha$ is finite.

Proof. Choose $s>0$ such that $\frac{1}{3} \leqq s<r$ and $2 s /(1-s)>2 r /(1-r)-\varepsilon$. Now there is a collection of $\alpha$ disjoint balls of radius $s$ in $U(X)$. Choose the set $B$ with card $(B)$ $=\alpha$ such that $\{y+s U(X) \mid y \in B\}$ is such a collection of balls. Since at most one of these balls contains the origin, the set $B^{\prime}=\{y \in B \mid\|y\| \geqq s\}$ has card $\left(B^{\prime}\right)=\alpha$ if $\alpha$ is infinite and card $\left(B^{\prime}\right) \geqq \alpha-1$ if $\alpha$ is finite. We first show that if the balls of radius $s$ centered at points of $B^{\prime}$ are pushed away from the origin until their centers have norm $1-s$ they remain disjoint. Let $x, y \in B^{\prime}$. Then

$$
s \leqq\|x\| \leqq 1-s, \quad s \leqq\|y\| \leqq 1-s \text { and }\|x-y\| \geqq 2 s
$$

and we wish to show

$$
\|(1-s) x /\| x\|-(1-s) y /\| y\|\| \geqq 2 s .
$$

Since $s \geqq \frac{1}{3}$ it follows from (1) that

$$
\|x\| \leqq \frac{2}{3} \leqq\|x-y\|
$$

and that there is a number $t, 0 \leqq t<1$, such that

$$
y=(1-t)(1-s) y /\|y\| \text {. }
$$

Thus

$$
x-y=t x+(1-t)[x-(1-s) y /\|y\|]
$$

Hence

$$
\|x-y\| \leqq t\|x\|+(1-t)\|x-(1-s) y /\| y\|\|
$$

or

$$
t\|x-y\|+(1-t)\|x-y\| \leqq t\|x\|+(1-t)\|x-(1-s) y /\| y\|\| .
$$

Applying (3) one obtains

$$
(1-t)\|x-y\| \leqq(1-t)\|x-(1-s) y /\| y\|\|
$$

or

$$
\|x-(1-s) y /\| y\|\| \geqq\|x-y\| \geqq 2 s .
$$

One proves (2) from (4) by repeating the above argument, replacing $x$ by $(1-s) y /\|y\|$ and replacing $y$ by $x$. Hence the set of points $\left\{(1-s) y /\|y\| \mid y \in B^{\prime}\right\}$ is a $2 s$-separated set. So the set $\left\{y /\|y\| \mid y \in B^{\prime}\right\}$ is a $2 s /(1-s)$-separated subset of $S(X)$ and

$$
2 s /(1-s)>2 r /(1-r)-\varepsilon \text { Q.E.D. }
$$


REMARK 1.4. Theorem 1.3 may be improved slightly in case $\alpha$ is finite and $r=\frac{1}{2}$. In this case, for each $\varepsilon>0$ there is a $(2-\varepsilon)$-separated subset $A$ of $S(X)$ with card $(A)$ $=\alpha$. The proof hinges on the fact that there exist $\alpha$ balls of radius arbitrarily close to $\frac{1}{2}$ interior to $U(X)$, none of which contain the origin. This fact is easy to verify and is left for the reader.

For an infinite dimensional normed linear space $X$. Lemma 1.2 shows that $P\left(\aleph_{0}, X\right)$ is a number in the interval $\left[\frac{1}{3}, \frac{1}{2}\right]$. We now present an example which proves a partial converse, that is, for each number $r \in\left(\frac{1}{3}, \frac{1}{2}\right]$ there is a Banach space $X$ with $P\left(\aleph_{0}, X\right)=r$. It is not known whether there is a normed space $X$ with $P\left(\aleph_{0}, X\right)=\frac{1}{3}$. In the following, the symbol $l_{p}$ denotes the usual space of $p$-summable sequences of real numbers.

Lemma 1.5. For $1 \leqq p<\infty$ and $\varepsilon>0$ there does not exist a $\left(2^{1 / p}+\varepsilon\right)$-separated subset of $S\left(l_{p}\right)$ of cardinality $\aleph_{0}$.

Proof. Suppose to the contrary that $\left\{x_{i}\right\}_{i=1}^{\infty}$ is a $\left(2^{1 / p}+\varepsilon\right)$-separated subset of $S\left(l_{p}\right)$. Choose $\delta>0$ such that $\left\{2\left[1+\delta^{1 / p}\right]^{p}+\delta\right\}^{1 / p}<2^{1 / p}+\varepsilon$. Let $z_{1}=x_{1}$ and $\left\{y_{i}^{1}\right\}_{i=1}^{\infty}$ $=\left\{x_{i}\right\}_{i=2}^{\infty}$ and $M_{1}=0$. Suppose by induction that $\left\{z_{1}, z_{2}, \ldots, z_{k-1}\right\}$ and a subsequence $\left\{y_{i}^{k-1}\right\}_{i=1}^{\infty}$ of $\left\{x_{i}\right\}_{i=1}^{\infty}$ and an integer $M_{k-1}$ have been chosen. Suppose that $z_{k-1}$ $=\left(a_{1}, a_{2}, \ldots\right)$. Choose $M_{k}>M_{k-1}$ so that $\sum_{i=M_{k}+1}^{\infty}\left|a_{i}\right|^{p}<\delta$. Choose a subsequence $\left\{w_{i}\right\}_{i=1}^{\infty}$ of $\left\{y_{i}^{k-1}\right\}_{i=1}^{\infty}$ such that $\left\{w_{i}\right\}_{i=1}^{\infty}$ converges coordinatewise on the first $M_{k}$ coordinates. Pick a positive integer $N$ so large that if $s, t \geqq N$ and $w_{s}=\left(c_{1}, c_{2}, \ldots\right)$ and $w_{t}=\left(d_{1}, d_{2}, \ldots\right)$ then $\sum_{i=1}^{M_{k}}\left|c_{i}-d_{i}\right|^{p}<\delta$. Define $z_{k}=w_{N}$ and $\left\{y_{i}^{k}\right\}_{i=1}^{\infty}=\left\{w_{i}\right\}_{i=N+1}^{\infty}$. In this way we obtain a subsequence $\left\{z_{1}, z_{2}, z_{3}, \ldots\right\}$ of $\left\{x_{i}\right\}_{i=1}^{\infty}$. Now, for each $y_{n}^{k-1}=\left(b_{1}, b_{2}, \ldots\right)$ we have $\sum_{i=M_{k-1}+1}^{M_{k}}\left|b_{i}\right|^{p} \geqq \delta$, for otherwise we would have

$$
\begin{aligned}
\left\|z_{k-1}-y_{n}^{k-1}\right\|^{p}= & \sum_{i=1}^{\infty}\left|a_{i}-b_{i}\right|^{p} \\
= & \sum_{i=1}^{M_{k}-1}\left|a_{i}-b_{i}\right|^{p}+\sum_{i=M_{k-1}+1}^{M_{k}}\left|a_{i}-b_{i}\right|^{p}+\sum_{i=M_{k}+1}^{\infty}\left|a_{i}-b_{i}\right|^{p} \\
\leqq & \sum_{i=1}^{M_{k}-1}\left|a_{i}-b_{i}\right|^{p} \\
& +\left[\left(\sum_{i=M_{k-1}+1}^{M_{k}}\left|a_{i}\right|^{p}\right)^{1 / p}+\left(\sum_{i=M_{k-1}+1}^{M_{k}}\left|b_{i}\right|^{p}\right)^{1 / p}\right]^{p} \\
& +\left[\left(\sum_{i=M_{k}+1}^{\infty}\left|a_{i}\right|^{p}\right)^{1 / p}+\left(\sum_{i=M_{k}+1}^{\infty}\left|b_{i}\right|^{p}\right)^{1 / p}\right]^{p} \\
\leqq & \delta+\left[1+\delta^{1 / p}\right]^{p}+\left[1+\delta^{1 / p}\right]^{p}
\end{aligned}
$$

from which it would follow $\left\|z_{k-1}-y_{n}^{k-1}\right\|<2^{1 / p}+\varepsilon$, a contradiction. But on the other hand, $\sum_{i=M_{h-1}+1}^{M_{k}}\left|b_{i}\right|^{p} \geqq \delta$ implies that $\left\|z_{n}\right\| \geqq(n \delta)^{1 / p}$ which for $n$ sufficiently large contradicts the fact that each $z_{n}$ has unit norm. Q.E.D.

EXAMPLE 1.6. For $1 \leqq p<\infty, P\left(\aleph_{0}, l_{p}\right)=2^{1 / p} /\left(2+2^{1 / p}\right)$. 
Proof. In $l_{p}$, let $e_{n}=(0, \ldots, 0,1,0, \ldots)$ where the 1 occurs in the $n$th coordinate. Now for each $\varepsilon>0$, the balls centered at $2 e_{n} /\left(2+2^{1 / p}\right)$ with radius $\left[2^{1 / p} /\left(2+2^{1 / p}\right)\right]-\varepsilon$ are disjoint and are in $U\left(l_{p}\right)$. Hence $P\left(\aleph_{0}, l_{p}\right) \geqq 2^{1 / p} /\left(2+2^{1 / p}\right)$. On the other hand, suppose that $P\left(\aleph_{0}, l_{p}\right)>2^{1 / p} /\left(2+2^{1 / p}\right)$. Then by Theorem 1.3 for $\delta$ small enough, $\delta>0$, there is a $\left(2^{1 / p}+\delta\right)$-separated subset of $S\left(l_{p}\right)$ of cardinality $\boldsymbol{\aleph}_{0}$. But by Lemma 1.5 this is impossible. Therefore $P\left(\aleph_{0}, l_{p}\right)=2^{1 / p} /\left(2+2^{1 / p}\right)$. Q.E.D.

REMARK 1.7. Let $\left\{B_{i}\right\}_{i=1}^{\infty}$ be a sequence of finite dimensional normed spaces and $p$ be a real number such that $1 \leqq p<\infty$. Define $X$ to be the $p$-product, $X=\mathscr{P}^{p}\left\{B_{i}\right\}$ (see, for example, $[1$, p. 314]). Then very gentle modifications of Lemma 1.5 and Example 1.6 show that $P\left(\aleph_{0}, X\right)=2^{1 / p} /\left(2+2^{1 / p}\right)$.

EXAMPLE 1.8. If $B_{i}$ represents the $i$-dimensional Banach space with the sup-norm for each positive integer $i$, and $X=\mathscr{P}^{p}\left\{B_{i}\right\}$ as in Remark 1.7, for some fixed $p$ with $1 \leqq p<\infty$, then for each positive integer $n, P(n, X)=\frac{1}{2}$ since $B_{n}$ is imbedded in $X$. On the other hand, by Remark 1.7, we have $P\left(\aleph_{0}, X\right)=2^{1 / p} /\left(2+2^{1 / p}\right)$. Hence it is false in general that $\lim _{n \rightarrow \infty} P(n, X)=P\left(\boldsymbol{\aleph}_{0}, X\right)$.

The conclusion of Example 1.8 may also be obtained from a theorem of Dvoretzky. His theorem [2, p. 124] implies that every infinite dimensional normed space has subspaces of arbitrarily high finite dimension whose norms are nearly spherical. Thus for every infinite dimensional normed space $X$ and positive integer $n, P(n, X) \geqq P\left(n, l_{2}\right) \geqq 2^{1 / 2} /\left(2+2^{1 / 2}\right)$, whereas $P\left(\aleph_{0}, l_{p}\right)<2^{1 / 2} /\left(2+2^{1 / 2}\right)$ for $p>2$.

2. Nearly isometric spaces. We show in this section that if the numbers $P(\alpha, X)$ and $P(\alpha, Y)$ differ for some Banach spaces $X$ and $Y$ and some cardinal number $\alpha$, then $X$ and $Y$ cannot be nearly isometric. Actually, a somewhat stronger result is obtained: we find a lower bound for the product $\|L\| \cdot\left\|L^{-1}\right\|$, where $L$ is a continuous isomorphism of $X$ onto $Y$, which is greater than one whenever $P(\alpha, X)$ $\neq P(\alpha, Y)$.

THEOREM 2.1. If for some cardinal number $\alpha$ and Banach spaces $X$ and $Y$, $P(\alpha, X) \neq P(\alpha, Y)$ then $X$ and $Y$ are not nearly isometric.

Proof. Suppose without loss of generality that $P(\alpha, X)>P(\alpha, Y)$. We will show that for any continuous isomorphism $L$ of $X$ onto $Y,\|L\| \cdot\left\|L^{-1}\right\| \geqq P(\alpha, X) / P(\alpha, Y)$. (Since density character is invariant under continuous isomorphisms onto, and (as Lemma 1.2 shows) $P(\alpha, Y)=0$ if and only if $\alpha$ is greater than the density character of $Y$, the theorem would follow immediately if $P(\alpha, Y)=0$.) To do this, choose $\delta$ such that $0<\delta<P(\alpha, X)$ and find a set $A$ such that $A$ is the set of centers of $\alpha$ disjoint balls of radius $P(\alpha, X)-\delta$ inside of $U(X)$. The set $B=\left\{\left\|L^{-1}\right\| \cdot L(a) \mid a \in A\right\}$ is a $2(P(\alpha, X)-\delta)$-separated set in $Y$. Furthermore, points in $B$ are bounded in norm by $\left\|L^{-1}\right\| \cdot\|L\|(1-P(\alpha, X)+\delta)$. Hence the balls of radius $P(\alpha, X)-\delta$ centered at points of $B$ are disjoint and are interior to

$$
\left[\left\|L^{-1}\right\| \cdot\|L\|(1-P(\alpha, X)+\delta)+(P(\alpha, X)-\delta)\right] U(Y) .
$$


But $\left\|L^{-1}\right\| \cdot\|L\|-\left\|L^{-1}\right\| \cdot\|L\|(P(\alpha, X)+\delta)+(P(\alpha, X)-\delta) \leqq\left\|L^{-1}\right\| \cdot\|L\|$. So multiplying by the reciprocal of $\left\|L^{-1}\right\| \cdot\|L\|$ we obtain $\alpha$ disjoint balls of radius

$$
(P(\alpha, X)-\delta) /\left\|L^{-1}\right\| \cdot\|L\|
$$

interior to $U(Y)$. Taking the infimum over $\delta$ we obtain $\|L\| \cdot\left\|L^{-1}\right\| \geqq P(\alpha, X) / P(\alpha, Y)$. Q.E.D.

3. Packing and reflexivity. A Banach space $X$ which is isometric to its second conjugate space $X^{\prime \prime}$ under the natural mapping (which assigns to each element $x$ of $X$ the linear functional $F_{x}$ defined by $F_{x}(f)=f(x)$ for each $f$ of $X^{\prime}$ ) is called reflexive. A Banach space is called uniformly non- $l_{n}^{(1)}$ provided there is a number $\varepsilon>0$ such that for each $n$ elements $\left\{x_{1}, \ldots, x_{n}\right\}$ of $S(X)$ it is true that

$$
(1 / n)\left\|x_{1} \pm x_{2} \pm \cdots \pm x_{n}\right\| \leqq 1-\varepsilon
$$

for some choice of signs. Geometrically, a uniformly non- $l_{n}^{(1)}$ space is one which does not have $n$-dimensional subspaces whose norms are arbitrarily good approximations of the $l_{1}$ norm. Daniel Giesy [3] and R. C. James [5] have raised the question whether Banach spaces which are uniformly non- $l_{n}^{(1)}$ for some positive integer $n \geqq 2$ (such spaces are called $B$-convex) are reflexive. James, in the paper cited above, settled the question affirmatively in the case $n=2$ and gave a partial result for the case $n=3$, but the general question remains unanswered. It is natural to ask whether reflexivity is implied by some stronger geometric condition.

Notice that a normed space $X$ which admits subspaces which are arbitrarily good approximations of $n$-dimensional $l_{1}$ space has $P(2 n, X)=\frac{1}{2}$. (To see this, consider the balls of radius $\frac{1}{2}-\varepsilon$ centered at the $2 n$ points of the form $(0, \ldots, 0$, $\left.\pm \frac{1}{2}, 0, \ldots, 0\right)$ in $n$-dimensional $l_{1}$ space.) Thus the requirement that $X$ satisfy $P(n, X)<\frac{1}{2}$ for some positive integer $n$ is a stronger condition on $X$ than that $X$ be uniformly non- $l_{n}^{(1)}$ for some integer $n>2$.

Definition 3.1. A Banach space $X$ is $P$-convex if $P(n, X)<\frac{1}{2}$ for some positive integer $\boldsymbol{n}$.

We have just seen that $P$-convexity implies $B$-convexity, and we now show that this stronger condition does imply reflexivity.

TheOREM 3.2. If a Banach space $X$ is P-convex, then it is reflexive.

Proof. We will prove that if $X$ is not reflexive, $n$ is a positive integer, and $\eta>0$, then there are $n$ balls of radius $\frac{1}{2}-\eta$ in $U(X)$ and hence $X$ is not $P$-convex.

The proof is similar to one of R. C. James [5, proof of Theorem 1.1, p. 543] and we will indicate here only how the present proof differs from that one. Let $\delta$ in that proof equal $\eta / n$, and after line (3) on p. 544 of that proof choose positive integers

$$
p_{1}^{1}, p_{2}^{1}, \ldots, p_{2 m}^{1} ; p_{1}^{2}, p_{2}^{2}, \ldots, p_{2 m}^{2} ; \ldots ; p_{1}^{n}, p_{2}^{n}, \ldots, p_{2 m}^{n}
$$


successively in the order

$\left\{p_{1}^{1}, p_{1}^{2}, \ldots, p_{1}^{n}, p_{2}^{1}, p_{3}^{1}, p_{2}^{2}, p_{3}^{2}, \ldots, p_{2}^{n}, p_{3}^{n}, p_{4}^{1}, p_{5}^{1}, p_{4}^{2}, p_{5}^{2}, \ldots\right.$

$$
\left.\ldots, p_{2 m-2}^{n}, p_{2 m-1}^{n}, p_{2 m}^{1}, p_{2 m}^{2}, \ldots, p_{2 m}^{n}\right\}
$$

so that this ordering also is by increasing magnitude. One may obtain in this manner $n$ increasing sequences $\left\{p_{1}^{1}, p_{2}^{1}, \ldots, p_{2 m}^{1}\right\}, \ldots,\left\{p_{1}^{n}, p_{2}^{n}, \ldots, p_{2 m}^{n}\right\}$ such that $S\left(p_{1}^{j}, p_{2}^{j}, \ldots, p_{2 m}^{j} ;\left\{f_{i}\right\}\right)$ has an element $u_{j}$ such that

(i) $\left\|u_{j}\right\| \leqq K\left(m,\left\{f_{i}\right\}\right)+\varepsilon$,

(ii) $K\left(m-1,\left\{f_{i}\right\}\right)-\varepsilon<\|z\|$ if

$$
z \in S\left(p_{3}^{j}, p_{2}^{j+1}, p_{5}^{j}, p_{4}^{j+1}, \ldots, p_{2 m-1}^{j}, p_{2 m-2}^{j+1} ;\left\{f_{i}\right\}\right)
$$

for all $j$ with $1 \leqq j \leqq n-1$. Now

$$
(1 / n)\left(-u_{1}-u_{2}-\cdots-u_{j}+u_{j+1}+\cdots+u_{n}\right)
$$

is an element of

$$
S\left(p_{3}^{j}, p_{2}^{j+1}, p_{5}^{j}, p_{4}^{j+1}, p_{7}^{j}, p_{6}^{j+1}, \ldots, p_{2 m-1}^{j}, p_{2 m-2}^{j+1} ;\left\{f_{i}\right\}\right)
$$

so by (ii) we have

$$
\left\|(1 / n)\left(-u_{1}-u_{2}-\cdots-u_{j}+u_{j+1}+\cdots+u_{n}\right)\right\| \geqq K\left(m-1,\left\{f_{i}\right\}\right)-\varepsilon
$$

for all $1 \leqq j \leqq n-1$. Let $y_{j}=u_{j} /\left(K_{m}+2 \varepsilon\right)$ for $j=1,2, \ldots, n$. By (3) of James' proof and (i) we have $\left\|y_{j}\right\| \leqq 1$ for all $j=1,2, \ldots, n$. And from (2) of James' proof and (5) it follows that

$$
\left\|\frac{1}{n}\left(-y_{1}-y_{2}-\cdots-y_{j}+y_{j+1}+\cdots+y_{n}\right)\right\| \geqq \frac{K_{m-1}-\varepsilon}{K_{m}+2 \varepsilon} \geqq 1-\delta
$$

for all $j=1,2, \ldots, n$. By the triangle inequality and (6) we obtain $\left\|y_{i}-y_{j}\right\| \geqq 2-n \delta$ $=2-\eta$ for all $1 \leqq i, j \leqq n$ with $i \neq j$. Finally, letting $x_{i}=y_{i} / 2$, it is clear that the balls of radius $\frac{1}{2}-\eta$ centered at the points of $\left\{x_{1}, x_{2}, \ldots, x_{n}\right\}$ are disjoint and are in $U(X)$. Q.E.D.

EXAMPLE 3.3. If $X$ represents the Banach space introduced by James [4] which is not reflexive but is isometric to its second conjugate space, one may show by methods similar to Lemma 1.5 and Theorem 1.6 that $P\left(\aleph_{0}, X\right)<\frac{1}{2}$. Therefore, Theorem 3.2 does not hold if the $n$ in the definition of $P$-convex is allowed to assume infinite values.

A Banach space is said to be uniformly convex if for each $\varepsilon>0$, there is $\delta(\varepsilon)>0$ such that whenever $x$ and $y$ are elements of $S(X),\|x-y\|>\varepsilon$ implies $\left\|\frac{1}{2}(x+y)\right\|$ $<1-\delta(\varepsilon)$. A Banach space is said to be uniformly smooth if for each $\varepsilon>0$ there is a $\delta(\varepsilon)>0$ such that whenever $x$ and $y$ are elements of $S(X),\|x-y\|<\delta(\varepsilon)$ implies $1-\left\|\frac{1}{2}(x+y)\right\| \leqq \varepsilon\|x-y\|$. It is known [1] that both uniform convexity and uniform smoothness imply reflexivity and that each property is the dual of the other, that is, if a Banach space $X$ is uniformly convex (smooth) then its dual $X^{\prime}$ is uniformly smooth (convex). 
One of the chief advantages of the concept of $B$-convexity is that it unifies the theories of uniformly smooth spaces and uniformly convex spaces, that is, $B$ convexity is weaker than both the other properties. This advantage is retained by $P$-convexity (and, as we shall see in the next section, by its dual property). The remainder of this section is devoted to proving this fact. We begin with two simple lemmas.

LEMMA 3.4. If $x$ and $y$ are elements of $S(X)$ such that $\|x-y\| \geqq 2-\varepsilon$ for some number $\varepsilon$ with $0 \leqq \varepsilon \leqq 1$, then $\|z\| \geqq 1-\varepsilon$ for each element $z$ of the line segment joining $x$ to $-y$.

Proof. Let $m$ be the midpoint of the line segment joining $x$ to $-y$. Then it follows immediately from the given that $\|m\| \geqq 1-\varepsilon / 2$. Let $z$ be an arbitrary point on the line segment between $x$ and $-y$ and suppose without loss of generality that $z$ lies between $x$ and $m$, that is, $\|x-z\|<\|z+y\|<\|x+y\| .(\|x+y\|$ is the distance from $x$ to $-y$.) Now suppose the lemma is false and $\|z\|<1-\varepsilon$. Then

$$
\begin{aligned}
\|m\|=\left\|\frac{1}{2}(x-y)\right\| & =\left\|-y+\frac{\|x+y\|}{2} \frac{z+y}{\|z+y\|}\right\| \\
& =\left\|\left(-1+\frac{\|x+y\|}{2\|z+y\|}\right) y+\frac{\|x+y\|}{2\|z+y\|} z\right\| \\
& <1-\frac{\|x+y\|}{2\|z+y\|}+\frac{\|x+y\|}{2\|z+y\|}(1-\varepsilon) \\
& =1-\frac{\|x+y\|}{2\|z+y\|} \varepsilon<1-\frac{\varepsilon}{2}
\end{aligned}
$$

which is a contradiction. Q.E.D.

LEMMA 3.5. If $P(3, X)=\frac{1}{2}$ then for each $\varepsilon>0$ there are three points $x, y$, and $z$ on $S(X)$ such that the three line segments $[x, y],[y, z]$, and $[z,-x]$ are all outside of $(1-\varepsilon) S(X)$.

Proof. By Remark 1.4, for each $\varepsilon>0$ there are three points $x, w$, and $z$ on $S(X)$ such that the distance between any two of them is at least $2-\varepsilon$. The conclusion of the lemma now follows immediately from Lemma 3.4, letting $y=-w$. Q.E.D.

TheOREM 3.6. $X$ is uniformly convex implies $P(3, X)<\frac{1}{2}$, and hence that $X$ is $P$-convex.

Proof. Suppose that $P(3, X)=\frac{1}{2}$ and $\delta=\delta\left(\frac{2}{3}\right)$ in the definition of uniformly convex. By Lemma 3.5 there are three line segments $[x, y],[y, z]$, and $[z,-x]$ whose end points are all on $S(X)$ and which are all outside of $(1-\delta) S(X)$. Since the distance from $x$ to $-x$ is 2 , one of these line segments must be at least as long as $\frac{2}{3}$, and this contradicts the uniform convexity. Q.E.D.

THEOREM 3.7. $X$ is uniformly smooth implies $P(3, X)<\frac{1}{2}$, and hence that $X$ is $P$-convex. 
Proof. $X$ is uniformly smooth implies the norm of $X$ is uniformly strongly (uniformly Fréchet) differentiable (see, for example, [8, p. 367]). This means that if $x \in S(X)$ and $x^{*} \in X^{\prime}$ such that $\left\|x^{*}\right\|=1$ and $x^{*}(x)=1$ then

$$
\lim _{t \rightarrow 0^{+}} \frac{\|x+t y\|-1}{t}=x^{*}(y)
$$

for all $y$ in $X$ and the limit is approached uniformly for all $x \in S(X)$ and all $y \in U(X)$. Now choose a positive number $\varepsilon$ such that $0<\varepsilon<1$ and find a positive number $t_{1}$ such that if $0<t \leqq t_{1}$ then

$$
\left|\frac{\|x+t y\|-1}{t}-x^{*}(y)\right|<\frac{\varepsilon}{4} \text { for all } x \in S(X) \text { and } y \in U(X) .
$$

Choose $\delta_{1}>0$ small enough that $\delta_{1} / t_{1}<\varepsilon / 4$ and find a positive number $t_{2}$ such that if $0<t \leqq t_{2}$ then

$$
\left|\frac{\|x+t y\|-1}{t}-x^{*}(y)\right|<\frac{\delta_{1}}{4} \text { for all } x \in S(X) \text { and } y \in U(X) .
$$

Finally, choose $\delta_{2}>0$ small enough that $\delta_{2} / t_{2}<\delta_{1} / 4$ and $\delta_{2}<\delta_{1}$. Now suppose the theorem is false and $P(3, X)=\frac{1}{2}$. Use $\delta_{2}$ in Lemma 3.5 to find three points $x, y$, and $z$ of $S(X)$ such that the line segments $[x, y],[y, z]$, and $[z,-x]$ are all outside of $\left(1-\delta_{2}\right) S(X)$. Let $y^{*}$ be the linear functional of $X^{\prime}$ of norm 1 such that $y^{*}(y)=1$. Now $(x-y) / 2 \in U(X)$, so by (2) we have

$$
\left|\left(|| y+t_{2}\left(\frac{x-y}{2}\right) \|-1\right) / t_{2}-y^{*}\left(\frac{x-y}{2}\right)\right|<\frac{\delta_{1}}{4}
$$

or

$$
\left|y^{*}\left(\frac{x-y}{2}\right)\right|<\frac{\delta_{1}}{4}+\left|\left(\left|y+t_{2}\left(\frac{x-y}{2}\right)\right| \mid-1\right) / t_{2}\right| .
$$

But the point $y+t_{2}(x-y) / 2$ is on the segment $[x, y]$ and hence has norm greater than $1-\delta_{2}$. Therefore the above inequality reduces to

$$
\left|y^{*}\left(\frac{x-y}{2}\right)\right|<\frac{\delta_{1}}{4}+\frac{\delta_{2}}{t_{2}}<\frac{\delta_{1}}{2}
$$

Therefore $\left|y^{*}(x-y)\right|<\delta_{1}$ and one may show by the same argument, replacing $x$ by $z$, that $\left|y^{*}(z-y)\right|<\delta_{1}$. Therefore $y^{*}(x)>1-\delta_{1}$ and $y^{*}(z)>1-\delta_{1}$ and therefore every point on the line segment $[x, z]$ has norm greater than $1-\delta_{1}$. Also, since $\delta_{1}>\delta_{2}$ every point on the line segment $[z,-x]$ has norm greater than $1-\delta_{1}$. Let $z^{*}$ be the linear functional of $X^{\prime}$ of norm 1 such that $z^{*}(z)=1$. Now $(x-z) / 2$ $\in U(X)$ so by (1) we have

$$
\left|\left(\left\|z+t_{1}\left(\frac{x-z}{2}\right)\right\|-1\right) / t_{1}-z^{*}\left(\frac{x-y}{2}\right)\right|<\frac{\varepsilon}{4}
$$


which reduces (using the same method as before) to $z^{*}(x)>1-\varepsilon$. Repeating this last step, replacing $x$ by $-x$ shows $z^{*}(-x)>1-\varepsilon$. But the last two inequalities form a contradiction, thus $P(3, X)<\frac{1}{2}$. Q.E.D.

4. The property dual to $P$-convexity. In the light of Theorem 3.2, the property dual to $P$-convexity becomes interesting and important, since it also implies reflexivity in Banach spaces. This section is devoted to specifying that property.

Definition 4.1. For $\varepsilon>0$, a convex subset $A$ of $U(X)$ is called an $\varepsilon$-flat if $A \cap(1-\varepsilon) U(X)=\varnothing$. A collection $\mathscr{D}$ of $\varepsilon$-flats is called complemented if, for each pair of $\varepsilon$-flats, $A$ and $B$, in $\mathscr{D}, A \cup B$ contains a pair of antipodal points. For each Banach space $X$ and cardinal number $\alpha$ define

$F(\alpha, X)$

$=\inf \{\varepsilon \mid U(X)$ contains a complemented collection of $\varepsilon$-flats of cardinality $\alpha\}$.

We now show that $P$-convexity is dual to the property that for some positive integer $n, F(n, X)>0$,

THEOREM 4.2. If $n$ is a positive integer and $\alpha$ is an arbitrary cardinal number, then

(a) $F\left(n, X^{\prime}\right)=0 \Rightarrow P(n, X)=\frac{1}{2}$,

(b) $P\left(n, X^{\prime}\right)=\frac{1}{2} \Rightarrow F(n, X)=0$,

(c) $F(\alpha, X)=0 \Rightarrow P\left(\alpha, X^{\prime}\right)=\frac{1}{2}$,

(d) $P(\alpha, X)=\frac{1}{2} \Rightarrow F\left(\alpha, X^{\prime}\right)=0$.

Proof. (a) Fix $\varepsilon>0$ and let $\delta=\varepsilon / 4$. Let $F_{1}, F_{2}, \ldots, F_{n}$ be a complemented collection of $\delta$-flats in $U\left(X^{\prime}\right)$. For $1 \leqq i<j \leqq n$ let $f_{i j} \in F_{i}$ such that $-f_{i j} \in F_{j}$ and define $f_{j i}=-f_{i j}$. Notice that $1-\delta \leqq\left\|f_{i j}\right\| \leqq 1$. Choose linear functionals $\varphi_{1}, \varphi_{2}, \ldots$, $\varphi_{n} \in S\left(X^{\prime \prime}\right)$ by the separation theorem (see, for example, [6, p. 22]) so that $\varphi_{i}\left(F_{i}\right)$ $\subset(1-\delta, 1]$. Using a Helly theorem (see $\S 1$, p. 320 of [9]) there exist points $x_{1}, x_{2}$, $\ldots, x_{n}$ in $X$ such that $\left\|x_{i}\right\|<1+\delta$ and $f_{j k}\left(x_{i}\right)=\varphi_{i}\left(f_{j k}\right)$ for all $j, k=1, \ldots, n$ with $j \neq k$. Now we have

$$
\begin{aligned}
\left\|x_{i}-x_{j}\right\| & \geqq \frac{1}{\left\|f_{i j}\right\|} f_{i j}\left(x_{i}-x_{j}\right) \\
& \geqq f_{i j}\left(x_{i}\right)-f_{i j}\left(x_{j}\right) \\
& =\varphi_{i}\left(f_{i j}\right)+\varphi_{j}\left(f_{j i}\right) \geqq 1-\delta+1-\delta .
\end{aligned}
$$

So $\left\|x_{i}-x_{j}\right\| \geqq 2-2 \delta$. Therefore

$$
\begin{aligned}
\left\|\frac{x_{i}}{\left\|x_{i}\right\|}-\frac{x_{j}}{\left\|x_{j}\right\|}\right\| & \geqq\left\|x_{i}-x_{j}\right\|-\left\|\frac{x_{i}}{\left\|x_{i}\right\|}-x_{i}\right\|-\left\|\frac{x_{j}}{\left\|x_{j}\right\|}-x_{j}\right\| \\
& \geqq 2-2 \delta-\delta-\delta=2-4 \delta=2-\varepsilon .
\end{aligned}
$$

Hence $S(X)$ admits a $(2-\varepsilon)$-separated subset of cardinality $n$. Shrinking by $\frac{1}{2}$ and taking the infimum over $\varepsilon$, we obtain $P(n, X)=\frac{1}{2}$. 
(b) Fix $\varepsilon>0$ and let $\delta$ be so small that $(1-2 \delta) /(1+\delta)>1-\varepsilon$. Since $P\left(n, X^{\prime}\right)=\frac{1}{2}$, by Remark 1.4 we may find $n$ points $f_{1}, f_{2}, \ldots, f_{n}$ on $S\left(X^{\prime}\right)$ such that $\left\|f_{i}-f_{j}\right\| \geqq 2-\delta$ for $i \neq j$. Let $L_{i j}$ be the line segment from $f_{i}$ to $-f_{j}$ for $i \neq j$, and define $m_{i j}$ to be the midpoint of $L_{i j}$. Notice that $\left\|m_{i j}\right\| \geqq 1-\delta$. Choose $\varphi_{i j}$ on $S\left(X^{\prime \prime}\right)$ by the HahnBanach theorem so that $\varphi_{i j}\left(m_{i j}\right)=\left\|m_{i j}\right\|$ and $\varphi_{i j}=-\varphi_{j i}$. It follows that $\varphi_{i j}\left(f_{i}\right) \geqq 1-2 \delta$. By the same Helly theorem as in part (a), choose $x_{i j}$ in $X$ such that $\left\|x_{i j}\right\| \leqq 1+\delta$ and $\varphi_{i j}\left(f_{k}\right)=f_{k}\left(x_{i j}\right)$ and $x_{i j}=-x_{j i}$ for all $i, j=1, \ldots, n$ with $i \neq j$ and all $k=1,2$, $\ldots, n$. Define $E_{i}=$ convex hull $\left(\left\{x_{i j}\right\}_{j=1}^{n}, j \neq i\right)$ and let $F_{i}=E_{i} /(1+\delta)$. Now $F_{i} \subset U(X)$ and $F_{i}$ is an $\varepsilon$-flat because

$$
\inf f_{i}\left(F_{i}\right) \geqq \operatorname{minimum}_{j=1, \ldots, n ; j \neq i} \frac{f_{i}\left(x_{i j}\right)}{1+\delta} \geqq \frac{1-2 \delta}{1+\delta}>1-\varepsilon .
$$

And the collection $\left\{F_{1}, \ldots, F_{n}\right\}$ is complemented since if $i \neq j$ then $x_{i j} /(1+\delta) \in F_{i}$ while $-x_{i j} /(1+\delta)=x_{j i} /(1+\delta) \in F_{j}$. The proofs of parts (c) and (d) are wholly analogous to those of parts (a) and (b) with the exception that since the Helly theorem is not needed, there is no restriction to the finite case. Q.E.D.

EXAMPLE 4.3. It is easy to verify that $P\left(\aleph_{1}, l_{\infty}\right)=\frac{1}{2}$. If parts (a) and (b) of Theorem 4.2 hold for arbitrary cardinal numbers, then (b) would imply that $F\left(\aleph_{1}, l_{1}\right)=0$ and (a) would imply that $P\left(\aleph_{1}, c_{0}\right)=\frac{1}{2}$ which is impossible since $c_{0}$ is separable. Hence some restriction on the cardinal number in parts (a) and (b) is essential.

Corollary 4.4. (a) $X$ is uniformly convex implies $F(3, X)>0$.

(b) $X$ is uniformly smooth implies $F(3, X)>0$.

(c) If for some positive integer $n, F(n, X)>0$, then $X$ is reflexive.

Proof. These facts follow from Theorems 3.6, 3.7, 3.2, 4.2, and a simple duality argument.

\section{REFERENCES}

1. M. M. Day, Reflexive Banach spaces not isomorphic to uniformly convex spaces, Bull. Amer. Math. Soc. 47 (1941), 313-317. MR 2, 221.

2. Aryeh Dvoretzky, Some results on convex bodies and Banach spaces, Proc. Internat. Sympos. Linear Spaces (Jerusalem, 1960), Jerusalem Academic Press, Jerusalem and Pergamon Press, Oxford, 1961, pp. 123-160. MR 25 \#2518.

3. Daniel P. Giesy, On a convexity condition in normed linear spaces, Trans. Amer. Math. Soc. 125 (1966), 114-146. MR 34 \#4866.

4. R. C. James, A non-reflexive Banach space isometric with its second conjugate space, Proc. Nat. Acad. Sci. U. S. A. 37 (1951), 174-177. MR 13, 356.

5. — Uniformly non-square Banach spaces, Ann. of Math. (2) 80 (1964), 542-550. MR 30 \#4139.

6. J. L. Kelley and I. Namioka, Linear topological spaces, The University Series in Higher Math., Van Nostrand, Princeton, N. J., 1963. MR 29 \#3851.

7. A. N. Kolmogorov and V. M. Tihomirov, e-entropy and e-capacity of sets in functional spaces, Uspehi Mat. Nauk 14 (1959), no. 2 (86), 3-86; English transl., Amer. Math. Soc. Transl. (2) 17 (1961), 277-364. MR 22 \#2890; MR 23 \#A2031. 
8. Gottfried Köthe, Topologische lineare Räume. Vol. I, Die Grundlehren der math. Wissenschaften, Band 107, Springer-Verlag, Berlin and New York, 1966. MR 33 \#3069.

9. Vlastimil Pták, Biorthogonal systems and reflexivity of Banach spaces, Czechoslovak Math. J. 9 (84) (1959), 319-326. MR 22 \#891.

10. Robert Whitley, The size of the unit sphere, Canad. J. Math. 20 (1968), 450-455. MR 37 \#4576.

Louisiana State University,

BATON ROUGE, LOUISIANA 70803 